\title{
Edge-magic group labellings of countable graphs
}

\author{
Nicholas Cavenagh and Diana Combe \\ School of Mathematics and Statistics \\ The University of New South Wales \\ Sydney, NSW 2052 Australia \\ nickc@maths.unsw.edu.au,diana@maths.unsw.edu.au
}

\author{
Adrian M. Nelson \\ School of Mathematics and Statistics \\ University of Sydney \\ NSW 2006 Australia \\ adriann@maths.usyd.edu.au
}

Submitted: May 18, 2006; Accepted: Sep 22, 2006; Published: Oct 27, 2006

Mathematics Subject Classification: 05C78

\begin{abstract}
We investigate the existence of edge-magic labellings of countably infinite graphs by abelian groups. We show for that for a large class of abelian groups, including the integers $\mathbb{Z}$, there is such a labelling whenever the graph has an infinite set of disjoint edges. A graph without an infinite set of disjoint edges must be some subgraph of $H+\mathcal{I}$, where $H$ is some finite graph and $\mathcal{I}$ is a countable set of isolated vertices. Using power series of rational functions, we show that any edge-magic $\mathbb{Z}$-labelling of $H+\mathcal{I}$ has almost all vertex labels making up pairs of half-modulus classes. We also classify all possible edge-magic $\mathbb{Z}$-labellings of $H+\mathcal{I}$ under the assumption that the vertices of the finite graph are labelled consecutively.
\end{abstract}

\section{Introduction.}

By countable we mean countably infinite. Our graphs have no loops and no multiple edges. The vertex set is non-empty and is denoted $V$. The edge set $E$ is a (possibly empty) set of unordered pairs of vertices. An edge $\{x, y\}$ is usually denoted $x y$ (or $y x$ ). The set $V \cup E$ is the set of graph elements. When we say a graph is countable we mean that the set of graph elements is countable, and hence that the vertex set is countable and the edge set is finite or countable. 
In this paper the group $\mathbb{A}$ is always a countable abelian group. Since we are often considering the integers, $\mathbb{Z}$, it is convenient to consider our groups additively. For a countable graph $G$, an $\mathbb{A}$-labelling of $G$, or a labelling of $G$ over $\mathbb{A}$, is a bijection from $V \cup E$ to $\mathbb{A}$. For any group, and any graph (finite or infinite) an injective labelling is an injection from the set of graph elements to the group.

Let $\lambda$ be a labelling of $G$ over a group $\mathbb{A}$. Then $\lambda$ defines a weight $\omega=\omega_{\lambda}$ on the edges. For $x y \in E$, the weight is the sum of the label of $x y$ and the labels of $x$ and $y$. That is,

$$
\omega(x y)=\lambda(x)+\lambda(x y)+\lambda(y)
$$

The labelling $\lambda$ is an edge-magic $\mathbb{A}$-labelling of $G$ if there is an element $k$ of $\mathbb{A}$ such that for every $x y \in E, \omega(x y)=k$. The element $k$ is the edge constant.

There are many different types of graph labellings which have been considered in recent years. Some are labelling the vertices, some the edges, and some, like those we are considering, are total labellings of all the graph elements. A detailed survey of many types of graph labellings can be found in the dynamic survey by Gallian [4]. The set of labels is commonly a subset of the integers, and a labelling can be used to define a weight on the edges (or the vertices). A magic labelling of a finite graph with $v$ vertices and $e$ edges is a total labelling of the graph by the integers $1,2,3, \ldots, v+e$ with constant edge (or vertex) weights. There is an extensive list of references about magic labellings of finite graphs in the book on Magic Graphs by Wallis [7]. Combe, Nelson and Palmer [2] generalised this to magic labellings of finite graphs, where the labels are the elements of an abelian group. This was extended to labellings of countably infinite graphs by countably infinite abelian groups by Beardon [1] and Combe and Nelson [3].

We are interested in this paper in edge-magic labellings of countable graphs by countable groups, and our emphasis is mainly on $\mathbb{Z}$-labellings. We are concerned with determining which graphs have labellings over which groups, what are the possible values of the magic constant, and also, since a total labelling of a graph partitions the group elements into vertex-labels and edge-labels, we are interested in properties of these partitions.

We denote by $\mathcal{I}$ the countable empty graph (a countable set of isolated vertices), by $K_{n}$ the complete graph with $n$ vertices, by $P_{n}$ the finite path of length $n$ and by $T_{n}$ the star with $n$ rays. If $H$ and $K$ are graphs, we denote by $H+K$ the join which is the graph which can be constructed by taking a copy of $H$ and a copy of $K$ (with no vertices in common) and adding all edges $\{h k: h \in H, k \in K\}$.

When a countable graph has an infinite set of independent edges, or has only finitely many edges, we show that there exists an edge-magic labelling over any countable subgroup of the additive real numbers (for example, the integers). A countable graph which does not contain an infinite set of independent edges is equal to a subgraph of $H+\mathcal{I}$ where $H$ is some finite graph (see Lemma 6). We call the graph $H+\mathcal{I}$ an $H$-burr and examine some examples for small $H$ in Section 3. In Section 4 we show that any an edgemagic $\mathbb{Z}$-labelling of an $H$-burr has almost all vertex labels lying in half-modulus classes (increasing or decreasing arithmetic progressions). In Section 5 we find exact necessary and sufficient conditions for an edge-magic $\mathbb{Z}$-labelling of an $H$-burr to exist for which the vertices of $H$ are labelled with consecutive integers. 


\section{Edge-magic labellings over $\mathbb{Z}$ and other groups.}

For a wide variety of countable graphs there are many edge-magic $\mathbb{Z}$-labellings, for example when there is an infinite set of mutually disjoint edges.

Theorem 1. Let $G$ be countable graph with an infinite set of mutually disjoint edges. Then, for any $k \in \mathbb{Z}$, there is an edge-magic $\mathbb{Z}$-labelling of $G$ with edge constant $k$.

Proof. Let $G$ be countable graph which has an infinite set of mutually disjoint edges and let $k \in \mathbb{Z}$. Fix a listing of the integers, $\mathbb{Z}=\left\{z_{1}, z_{2}, \ldots\right\}$. Fix a listing of the vertices, $V=\left\{v_{1}, v_{2}, \ldots\right\}$. Let $e_{1}, e_{2}, \ldots$ be an infinite sequence of mutually disjoint edges. Then for each $i, e_{i}=v_{\alpha_{i}} v_{\beta_{i}}$, where $\alpha_{i}<\beta_{i}$, and we define define $G_{i}$ to be the (finite) subgraph of $G$ induced by the vertices $\left\{v_{1}, v_{2}, \ldots, v_{\beta_{i}}\right\}$.

Step 1: Take the first edge, $e_{1}=v_{\alpha_{1}}, v_{\beta_{1}}$.

We define an injective map from the integers to the graph elements of $G_{1}$ which has a constant edge weight of $k$. Choose $a \in \mathbb{Z}, a \neq 0, \pm k$. Map $e_{1} \mapsto k, v_{\alpha_{1}} \mapsto a$ and $v_{\beta_{1}} \mapsto-a$. If $\beta_{1}>2$ then there are $\beta_{1}-2$ vertices in $G_{1}$ which are yet to be labelled. (Our plan is to label them with positive integers which are very much larger than any integer which has previously been used as a label. We choose labels for the vertices which are successively much larger than each other, so that they are distinct and so that the labels forced on any edges between them are all distinct.) Set $m=2+|k|+|a|+|-a|$. Label the $v_{\beta_{1}}-2$ vertices by the first $v_{\beta_{1}}-2$ terms of the sequence $2^{m}, 2^{2^{m}}, 2^{2^{2^{m}}} \ldots$. All vertices of $G_{1}$ are now labelled. If there is any edge in $G_{1}$ which is as yet unlabelled, label it with the unique integer which gives a constant edge weight of $k$. Observe that any integers which arise as edge labels are distinct from any labels already used, and that no two edges can require the same label.

Step 2: Let $\mathbf{L}$ denote the set of integers which have been used as labels so far. Take the first unlabelled edge in the sequence of mutually disjoint edges, $e_{j}$, say. Then we have $e_{j}=v_{\alpha_{j}}, v_{\beta_{j}}$. We extend the injective $\mathbb{Z}$-labelling of $G_{1}$ to an injective $\mathbb{Z}$-labelling of $G_{j}$ which defines a constant edge weight of $k$. Let $b_{j} \in \mathbb{Z}, b_{j} \notin \mathbf{L}$, be the first integer in the list which has not been used as a label. Map $e_{j} \mapsto b_{j}$. Set $m_{j}=2+\left|b_{j}\right|+\sum_{l \in \mathbf{L}}|l|$. Map $v_{\alpha_{j}} \mapsto 2^{m_{j}}$ and $v_{\beta_{j}} \mapsto k-b_{j}-2^{m_{j}}$. Now if $v_{\beta_{j}}>2+v_{\beta_{1}}$ then there are $v_{\beta_{j}}-v_{\beta_{1}}-2$ vertices in $G_{j}$ which are yet to be labelled. Label these by the first $v_{\beta_{j}}-v_{\beta_{1}}-2$ terms of the sequence $2^{2^{m_{j}}}, 2^{2^{2^{m_{j}}}}, \ldots$.

All vertices of $G_{j}$ are now labelled. Label any edges in $G_{j}$ which were not in $G_{1}$ with the unique integer which gives a constant edge weight of $k$.

How can we be sure that this is an injective labelling of $G_{j}$ ? To see this, set $\mathbf{M}=$ $\left\{2^{m_{j}}, 2^{2^{m_{j}}}, 2^{2^{2^{m_{j}}}}, \ldots\right\}$. Vertices are labelled with distinct elements of $\mathbf{L} \cup\left\{k-b_{j}-2^{m_{j}}\right\} \cup \mathbf{M}$, where $m_{j}>>|l|$ for any $l \in \mathbf{L}$ (including $k \in \mathbf{L}$ ). The labels on the additional edges in $G_{j}$ are all of the form of one of the following:

$$
\begin{aligned}
& k-x-y, \quad x \in \mathbf{L}, \quad y \in \mathbf{M}, \\
& k-y-z, \quad y, z \in \mathbf{M}, \quad y \neq z,
\end{aligned}
$$




$$
\begin{gathered}
2^{m_{j}}+b_{j}-x, \quad x \in \mathbf{L}, \\
2^{m_{j}}+b_{j}-y, \quad y \in \mathbf{M}, \quad y \neq 2^{m_{j}} .
\end{gathered}
$$

Note that for distinct $x \in \mathbf{L}$ and $y, z \in \mathbf{M}$, these integers are distinct and are distinct from any of the vertex labels. Therefore this is an injective $\mathbb{Z}$-labelling of $G_{j}$ which defines a constant edge weight of $k$.

Step $\mathbf{n}+\mathbf{1}$ : Let $\mathbf{L}$ denote the set of integers which have been used as labels so far. Take the next as-yet-unlabelled edge in the sequence of mutually disjoint edges. In the same manner as above extend the injective $\mathbb{Z}$-labelling.

Note that by step $n$ the vertices $v_{1}, v_{2}, \ldots v_{n}$ have been labelled and the integers $z_{1}, z_{2}, \ldots z_{n}$ have been used as labels. Therefore, this recursively defines an edge-magic $\mathbb{Z}$-labelling of $G$ which has constant edge weight of $k$.

In the proof above we could have had labels over the rational numbers, $\mathbb{Q}$, or any countable subgroup of the real numbers which included the number 2. Only a small modification of the proof is required for any countable subgroup of real numbers which does not include 2. For let $g$ be an element of the group such that $g \geq 2$. In the definition of $m$ and $m_{j}$, replace 2 with $g$, and replace the various expressions of the form $2^{m}, 2^{2^{m}}, 2^{2^{2^{m}}}, \ldots 2^{m_{j}}, 2^{2^{m_{j}}}, 2^{2^{2^{m_{j}}}}, \ldots$ with $g^{m}, g^{g^{m}}, g^{g^{g^{m}}}, \ldots g^{m_{j}}, g^{g^{m_{j}}}, g^{g^{g^{m_{j}}}}, \ldots$ Therefore it follows that:

Theorem 2. Let $G$ be countable graph which has an infinite set of mutually disjoint edges and let $\mathbb{A}$ be isomorphic to a countable subgroup of the additive real numbers. Let $k \in \mathbb{A}$. Then there is an edge-magic $\mathbb{A}$-labelling of $G$ which has edge constant $k$.

Note this includes any $\mathbb{A}$ which is a direct sum of a finite or countably many copies of $\mathbb{Z}$ or $\mathbb{Q}$, and more generally any countable torsion-free abelian group.

It is not the case that every countable graph which has an infinite set of mutually disjoint edges will necessarily have edge-magic labellings over an arbitrary countable group. For example the graph which consists precisely of an infinite set of mutually disjoint edges does not have any isolated vertices and the following result shows that it does not have edge-magic labellings over all countable groups.

Theorem 3. Let $G$ be a countable graph with no isolated vertices and let $\mathbb{A}$ be a countable group with $2 \mathbb{A}=\{0\}$, then there are no edge-magic $\mathbb{A}$-labellings of $G$.

Proof. Suppose there is an edge-magic $\mathbb{A}$-labelling of $G$ which has edge constant $k$. For each edge, we obtain an equation $a+b+c=k$, where $a, b$ and $c$ are (necessarily distinct) labels on the edge and its two end vertices. For at least one edge, one of these labels (say c) must equal $k$, so that $a+b+k=k$. But, for this group, this means $a=b$, which is a contradiction. Hence there are no edge-magic $\mathbb{A}$-labellings of $G$. 


\section{$3 \quad$ Edge-magic $\mathbb{Z}$-labellings of $H$-burrs.}

Definition 4. A countable graph $G$ is called a burr if it does not have an infinite set of mutually disjoint edges.

For example, if $G$ has only finitely many edges, then $G$ is a burr.

Theorem 5. If a countable graph $G$ has only finitely many edges, and $\mathbb{A}$ is isomorphic to a countable subgroup of the additive real numbers, then, for any $k \in \mathbb{A}$, there is an edge-magic $\mathbb{A}$-labelling of $G$ which has edge constant $k$.

Proof. In this case the edges involve only a finite set of the vertices, $v_{1}, v_{2}, \ldots, v_{N}$, say, and there are infinitely many isolated vertices. Here we can use a similar argument to that in Theorem 1. Label $v_{1}, v_{2}, \ldots, v_{N}$ with successively larger group elments, so that the labels forced on any edges are all distinct. Finally, label isolated vertices with the remaining group elements.

More generally, burrs can be characterised as follows:

Lemma 6. Suppose that $G$ is a burr. Then there is finite subgraph $H$ (possibly not unique) of $G$ such that all the edges of the graph have at least one vertex in $H$. That is, for some finite graph $H, G$ is (isomorphic to) a subgraph of $H+\mathcal{I}$.

Proof. Take a maximal set of disjoint edges of $G$. Let $V$ be the set of vertices incident to these edges and let $H$ be the subgraph induced by $V$.

Definition 7. Suppose that $G$ is a burr with a finite subgraph, $H$ say, such that all vertices outside $H$ have edges to all vertices inside $H$ and there are no edges between any vertices outside $H$. Then we say $G$ is an $H$-burr. Clearly a countable graph $G$ is an $H$-burr if (and only if) it has a finite subgraph $H$ such that $G \cong H+\mathcal{I}$.

For the remainder of the paper we are particularly interested in edge-magic $\mathbb{Z}$-labellings of $H$-burrs. We often use the following lemma from [3]:

Lemma 8. Let $G$ be a countable graph with an edge-magic $\mathbb{Z}$-labelling, then

(i) There is an edge-magic $\mathbb{Z}$-labelling of $G$ with edge constant $k=0$ or 1 .

(ii) If $G$ has an edge-magic $\mathbb{Z}$-labelling with edge constant $k=0$, then $G$ has an edgemagic $\mathbb{Z}$-labelling with constant $k$ for all $k \equiv 0 \bmod 3$.

(iii) If $G$ has an edge-magic $\mathbb{Z}$-labelling with constant $k=1$, then $G$ has an edge-magic $\mathbb{Z}$-labelling with edge constant $k$ for any $k \not \equiv 0 \bmod 3$. 


\subsection{Some examples: $P_{n}$-burrs and $K_{n}$-burrs.}

Example 9. A $P_{0}$-burr is just a countable star. From [3], when $G$ is a $P_{0}$-burr, then

(i) $G$ has an edge-magic $\mathbb{Z}$-labelling with constant $k$ if and only if $k \equiv 0 \bmod 3$.

(ii) $G$ has no edge-magic labellings over a group containing an element of order 2 .

Example 10. Let $G$ be a $P_{1}$-burr. Then for each $k \in \mathbb{Z}$ then there is an edge-magic $\mathbb{Z}$ labelling of $G$ with edge constant $k$. We can assume that $V=\left\{u, v, v_{1}, v_{2}, \ldots\right\}$ and $E=$ $\left\{u v, u v_{1}, v v_{1}, u v_{2}, v v_{2}, \ldots\right\}$. It is sufficient to find edge-magic labellings with constants 0 and 1. In each case we choose integers to label the elements of $P_{1}$ and extend this to a labelling of the $P_{1}$-burr.

(i) $k=0$. Define $\lambda(u)=1, \lambda(v)=-1, \lambda(u v)=0$. This can be extended in only one way to an edge-magic $\mathbb{Z}$-labelling of $G$ with constant $k=0$. For each $i \geq 1, \lambda\left(v_{2 i-1}\right)=$ $-3 i, \lambda\left(v_{2 i-1} u\right)=3 i-1, \lambda\left(v_{2 i-1} v\right)=3 i+1, \lambda\left(v_{2 i}\right)=3 i, \lambda\left(v_{2 i-1} u\right)=-3 i-1$ and $\lambda\left(v_{2 i} v\right)=-3 i+1$. Note that for $i=1,2, \ldots$, the set of labels on the vertices $\left\{u, v, v_{i}, \ldots v_{2 i}\right\}$ and all edges connecting them in $G$ is the set $\{0, \pm 1, \pm 2, \ldots, \pm 3 i\}$.
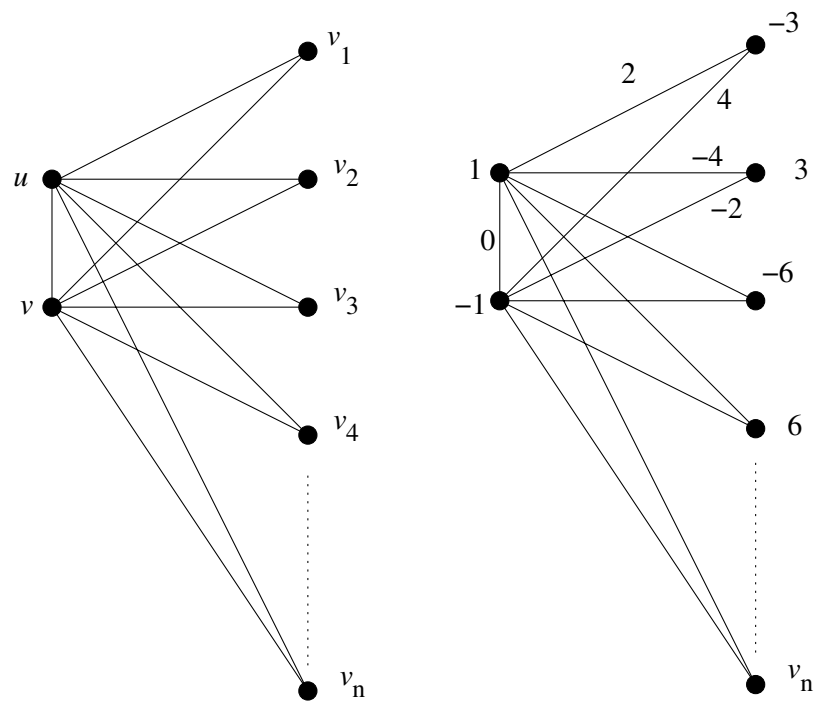

Figure 1: The $P_{1}$-burr

The labels of the vertices are

$$
\{ \pm 1\} \cup\{z \mid z<0, z \equiv 0 \bmod 3\} \cup\{z \mid z>0, z \equiv 0 \bmod 3\}
$$

This is a union of a finite set and two "half-modulus classes mod 3". 
Another solution is to define $\lambda(u)=0, \lambda(v)=-1, \lambda(u v)=1$. Then there is exactly of one way of extending this to an edge-magic $\mathbb{Z}$-labelling of $G$ with constant $k=0$. In this case the vertex labels are

$$
\{0,1\} \cup\{z \mid z \neq-1, z \equiv 2 \bmod 3\}
$$

(ii) $k=1$. Define $\lambda(u)=-1, \lambda(v)=2, \lambda(u v)=0$. There is only one way to extend this to an edge-magic $\mathbb{Z}$-labelling with $k=1$ : for $i=0,1,2, \ldots$

$$
\begin{array}{ccc}
\lambda\left(v_{6 i+1}\right)=-2-9 i, & \lambda\left(v_{6 i+1} u\right)=9 i+4, & \lambda\left(v_{6 i+1} v\right)=9 i+1 \\
\lambda\left(v_{6 i+2}\right)=-4-9 i, & \lambda\left(v_{6 i+2} u\right)=9 i+6, & \lambda\left(v_{6 i+2} v\right)=9 i+3 \\
\lambda\left(v_{6 i+3}\right)=5+9 i, & \lambda\left(v_{6 i+3} u\right)=-9 i-3, & \lambda\left(v_{6 i+3} v\right)=-9 i-6 \\
\lambda\left(v_{6 i+4}\right)=7+9 i, \quad \lambda\left(v_{6 i+4} u\right)=-9 i-5, & \lambda\left(v_{6 i+4} v\right)=-9 i-8 \\
\lambda\left(v_{6 i+5}\right)=9+9 i, \quad \lambda\left(v_{6 i+5} u\right)=-9 i-7, & \lambda\left(v_{6 i+5} v\right)=-9 i-10 \\
\lambda\left(v_{6 i+6}\right)=-9-9 i \quad \lambda\left(v_{6 i+6} u\right)=9 i+11, & \lambda\left(v_{6 i+6} v\right)=9 i+8 .
\end{array}
$$

It is straightforward to verify that $\lambda$ is injective. For $i=1,2, \ldots$, the set of labels on the vertices $\left\{u, v, v_{1}, \ldots v_{6 i}\right\}$ and all edges connecting them in the graph $G$ is the set $\{-(9 i+1), 9 i+2\} \cup\{0, \pm 1 \pm 2, \pm 3, \ldots, \pm 9 i\}$. So $\lambda$ is a surjection. It is immediate that $\lambda$ defines a constant edge weight of 1 . Note that the integers which are labels of the vertices are

$$
\{-1,2\} \cup\{z \mid z \equiv 7 \bmod 9\} \cup\{z \mid z \equiv 5 \quad \bmod 9\} \cup\{z \mid z \neq 0, z \equiv 0 \bmod 9\} .
$$

Another solution is to define $\lambda(u)=0, \lambda(v)=2, \lambda(u v)=-1$. Then there is exactly one way to extend this to an an edge-magic $\mathbb{Z}$-labelling of $G$. In this case the vertex labels are

$$
\{0,2\} \cup\{z \mid z \equiv 4 \bmod 6\} \cup\{z \mid z \neq-1, z \equiv 5 \bmod 6\}
$$

Note the choice of initial injective labelling of $P_{1}$ with constant edge weight is crucial. Suppose we begin begin by defining $\lambda(u)=0, \lambda(v)=-1, \lambda(u v)=2$. Then there is no way to extend this to an edge-magic $\mathbb{Z}$-labelling of $G$, for the label 1 can not be used.

Example 11. Let $G$ be $P_{2}$-burr. Then, for any $k \in \mathbb{Z}$, there is an edge-magic $\mathbb{Z}$-labelling of $G$ which has edge constant $k$.

Proof. Let $G$ be a $P_{2}$-burr, then we can assume that $V=\left\{u, v, w, v_{1}, v_{2}, v_{3}, \ldots\right\}$ and $E=\left\{u v, v w, u v_{1}, v v_{1}, w v_{1}, u v_{2}, v v_{2}, w v_{2}, \ldots\right\}$. It is sufficient to find edge-magic labellings with constants 0 and 1 . It is straightforward to show that the following define appropriate edge-magic labellings. 
(i) $k=0$.

Define $\lambda(u)=2, \lambda(v)=0, \lambda(w)=1, \lambda(u v)=-2, \lambda(v w)=-1$.

For $i=0,1,2, \ldots$, define $\lambda\left(v_{2 i-1}\right)=3+4 i$ and $\lambda\left(v_{2 i}\right)=-6-4 i$.

Label the edges to give a constant edge weight of $k=0$.

(ii) $k=1$.

Define $\lambda(u)=1, \lambda(v)=2, \lambda(w)=-1, \lambda(u v)=-2, \lambda(v w)=0$.

For $i=1,2, \ldots$, define $\lambda\left(v_{2 i-1}\right)=-4 i$ and $\lambda\left(v_{2 i}\right)=1+4 i$.

Label the edges to give a constant edge weight of $k=1$.

An edge-magic $\mathbb{Z}$-labelling of $P_{5}+\mathcal{I}$ is given later in Example 48. In general, the problem of determining an edge-magic $\mathbb{Z}$-labelling of $P_{n}+\mathcal{I}$ and $K_{n}+\mathcal{I}$ for arbitrary $n$ appears difficult. Even the case $K_{3}+\mathcal{I}$ remains open.

\section{Edge-magic integer labellings of $H$-burrs and half- modulus classes of vertex labels.}

Throughout this section we assume that $G$ is an $H$-burr. That is, $G=H+\mathcal{I}$ for a finite graph $H$ with $m$ vertices. If $m=1$ then $G$ is a countable star. However, stars were dealt with in [3]. So we are interested here in the case $m>1$. We assume that there is an edge-magic $\mathbb{Z}$-labelling of $G$. We introduce the following notation:

- $H_{V}$ denotes the set of labels of the Vertices of $H$.

- $H_{E}$ denotes the set of labels of the Edges of $H$.

- $B_{E}$ denotes the set of labels of the Edges Between $H$ and $\mathcal{I}$.

- $I_{V}$ denotes the set of labels of the Vertices of $\mathcal{I}$.

Thus $H_{V}, H_{E}, B_{E}$ and $I_{V}$ partition $\mathbb{Z}$, and $H_{E}$ is the only one of the sets which may be empty. Note that $H_{V}$ determines $H_{E}$, that $H_{V}$ and $I_{V}$ together determine $B_{E}$.

Let $d$ be a positive integer. A positive half-modulus class modulo $d$ is the set of terms of an arithmetic progression with common difference $d$. Similarly, a negative half-modulus class modulo $d$ is the set of terms of an arithmetic progression with common difference $-d$.

We are motivated in this section by considering the vertex labels which occur in an edge-magic $\mathbb{Z}$-labelling of $G$. In Example 10, $G=P_{1}+\mathcal{I}, m=2$ and $k=1$. The set of vertex labels is $\{-1,2\} \cup\{z>0 \mid z \equiv 7 \bmod 9\} \cup\{z<0 \mid z \equiv 7$ $\bmod 9\} \cup\{z>0 \mid z \equiv 5 \bmod 9\} \cup\{z<0 \mid z \equiv 5 \bmod 9\} \cup\{z>0 \mid z \equiv 0$ $\bmod 9\} \cup\{z<0 \mid z \equiv 0 \bmod 9\}$. Note that if we set $\mu=3$, then this is a union of a finite set and $2 \mu$ half-modulus classes $\bmod \mu \times(m+1)$.

We use the following result which can be found in Pólya and Szegö [6]: 
Theorem 12. The set of coefficients of a power series expansion of a rational function is finite if and only if the sequence of coefficients is eventually periodic.

We make the following observation:

Observation 13. If a power series $\sum f_{n} z^{n}$ has its series of coefficients periodic of period $M$ for $n \geq K$, i.e. $f_{K}, f_{K+1}, \ldots$ is periodic of period $M$, then

$$
\sum_{n=0}^{\infty} f_{n} z^{n}=f_{0}+f_{1} z+\cdots+f_{K-1} z^{K-1}+\frac{z^{k}\left(f_{K}+f_{K+1} z+\ldots f_{K+M-1} z^{M-1}\right)}{\left(1-z^{M}\right)} .
$$

Our main result in this section is:

Theorem 14. Let $G=H+\mathcal{I}$ be an $H$-burr which is not simply a star. That is $m$, the number of vertices in $H$, is greater than 1 . Suppose that there is an edge-magic $\mathbb{Z}$-labelling of $G$. Then, for some number $\mu$, the vertex labels of $G$ consist of a finite set and a disjoint union of $\mu$ positive half-modulus classes and $\mu$ negative half-modulus classes all modulo $\mu(m+1)$.

Proof. Our calculations take place in the quotient field $\mathbb{Q}((z))$ of the ring $\mathbb{Q}[[z]]$ of formal power series in $z$ with rational coefficients. For $f(z), g(z) \in \mathbb{Q}((z))$, define $f(z) \approx g(z)$ if $f(z)$ and $g(z)$ differ by a finite number of terms, that is if $f(z)-g(z) \in \mathbb{Q}\left[z, z^{-1}\right]$, where $\mathbb{Q}\left[z, z^{-1}\right]$ denotes the subring of $\mathbb{Q}((z))$ consisting of polynomials over $\mathbb{Q}$ in $z$ and $z^{-1}$.

Suppose that there is an edge-magic $\mathbb{Z}$-labelling of $G$ with edge-magic constant $k$. Consider the two power series in $\mathbb{Q}[[z]]$ defined using the labels on the vertices of $\mathcal{I}$ :

$$
p(z)=\sum_{x \geq 0, x \in I_{V}} z^{x}
$$

and

$$
q(z)=\sum_{x \leq 0, x \in I_{V}} z^{-x}
$$

Define an element of $\mathbb{Q}\left[z, z^{-1}\right]$ using the labels on the vertices of $H$ :

$$
a(z)=\sum_{a \in H_{V}} z^{a} .
$$

In general, for $\left.f(z) \in \mathbb{Q}\left[z, z^{-1}\right]\right)$, define $\hat{f}(z)=f(1 / z)$. In particular

$$
\hat{a}(z)=\sum_{a \in H_{V}} z^{-a} .
$$

Edges which are not in $H$ are edges from $H$ to $\mathcal{I}$. Their labels are the integers of the form $k-a-x$, with $a \in H_{V}$ and $x \in I_{V}$. Now, $k-a-x>0$ for all sufficiently large and negative $x$. Therefore,

$$
z^{k} \hat{a}(z) q(z) \approx \sum_{y \geq 0, y \in B_{E}} z^{y}
$$


Now every sufficiently large positive integer labels either an edge not in $H$ or a vertex not in $H$, and hence:

$$
z^{k} \hat{a}(z) q(z)+p(z) \approx \sum_{n=0}^{\infty} z^{n}=\frac{1}{1-z}
$$

Similarly, $k-a-x$ is negative for all sufficiently large positive $x$, hence

$$
z^{-k} a(z) p(z) \approx \sum_{y \leq 0, y \in B_{E}} z^{-y}
$$

and

$$
z^{-k} a(z) p(z)+q(z) \approx \frac{1}{1-z}
$$

From these equivalences we have that

$$
z^{k} \hat{a}(z) q(z)+p(z)=\frac{1}{1-z}+r(z)
$$

and

$$
z^{-k} a(z) p(z)+q(z)=\frac{1}{1-z}+s(z)
$$

where $s(z), r(z) \in \mathbb{Q}\left[z, z^{-1}\right]$.

This system of equations is linear in $q$ and $p$, with coefficients and right-hand-side expressions rational functions of $z$. When the determinant of the system

$$
\left|\begin{array}{cc}
z^{k} \hat{a}(z) & 1 \\
1 & z^{-k} a(z)
\end{array}\right|=a(z) \hat{a}(z)-1
$$

is non-zero we can solve this system for $p(z)$ and $q(z)$. Now $a(z) \hat{a}(z)=1$ if and only if $a(z)=z^{a}$ for some $a \in \mathbb{Z}$, which will not be the case for $m>1$. We deduce that, since the graph $H$ is not a countable star, $p(z)$ and $q(z)$ are rational functions of $z$.

Since all the coefficients of $p(z)$ and $q(z)$ are either 0 or 1 , we can apply Theorem 12 to both series. Therefore, for some positive integers $K$ and $M$ the power series $p(z)$ and $q(z)$ have their sequence of coefficients periodic of period $M$ for $n \geq K$. (Note that we can choose the same $K$ in both sequences, because if we had different $K^{\prime} s$ then the larger value of $K$ would be appropriate for each sequence, and if we had different $M^{\prime} s$ then the product of them would be an $M$ appropriate for each sequence.)

We now apply Observation 13 to $p(z)$, bearing in mind that the coefficients are each 0 or 1 , to deduce that there exist integers

$$
0 \leq b_{1}<b_{2}<b_{3}<\cdots<b_{\mu}<M
$$


such that

$$
p(z)=p_{0}+p_{1} z+\cdots+p_{K-1} z^{K-1}+z^{K}\left(\frac{z^{b_{1}}+\cdots+z^{b_{\mu}}}{1-z^{M}}\right) .
$$

The term

$$
z^{K}\left(\frac{z^{b_{1}}+\cdots+z^{b_{\mu}}}{1-z^{M}}\right)=\sum_{i=1}^{\mu}\left(\sum_{j=0}^{\infty} z^{K+b_{i}+M j}\right) .
$$

Thus Equation 19 tells us that an integer $x \geq K$ labels a vertex of $\mathcal{I}$ if and only if $x$ belongs to the union of arithmetic progressions with first term one of $b_{1}+K, b_{2}+K, \ldots, b_{\mu}+K$ and common difference $M$. Note that this is a disjoint union of half-modulus classes because the integers $b_{1}, \ldots b_{\mu}$ are distinct modulo $M$.

Similarly there exist integers

$$
0 \leq c_{1}<c_{2}<c_{3}<\cdots<c_{\nu}<M
$$

such that

$$
q(z)=q_{0}+q_{1} z+\cdots+q_{K-1} z^{K-1}+z^{K}\left(\frac{z^{c_{1}}+\cdots+z^{c_{\nu}}}{1-z^{M}}\right),
$$

which implies that the integers less than or equal to $-K$ which are labels of vertices of $\mathcal{I}$ consist of the negatives of the terms arithmetic progressions with first terms $K+c_{1}, K+$ $c_{2}, \cdots, K+c_{\nu}$ and common difference $M$.

To complete the proof of the theorem it remains to show $\nu=\mu$ and $M=\mu(m+1)$.

From (17) and $\frac{1}{1-z}=\frac{1+z+\cdots+z^{M-1}}{1-z^{M}}$ we deduce

$$
z^{k} \hat{a}(z) q(z)+p(z)=r(z)+\frac{1+z+\cdots+z^{M-1}}{1-z^{M}} .
$$

Set

$$
b(z)=z^{b_{1}}+\cdots+z^{b_{\mu}} \quad \text { and } \quad c(z)=z^{c_{1}}+\cdots+z^{c_{\nu}} .
$$

From (19) and (21) we deduce $\left(1-z^{M}\right) p(z)$ and $\left(1-z^{M}\right) q(z)$ are polynomials in $z$ congruent to $z^{K} b(z)$ and $z^{K} c(z)$ respectively. So if we multiply (22) through by $1-z^{M}$ we get an equation in $\mathbb{Q}\left[z, z^{-1}\right]$ which we can divide by $z^{K}$ and reduce modulo $1-z^{M}$ to deduce

$$
z^{k} \hat{a}(z) c(z)+b(z) \equiv 1+z+\cdots+z^{M-1} \quad \bmod \left(1-z^{M}\right) .
$$

Similarly from (18) follows

$$
z^{-k} a(z) b(z)+c(z) \equiv 1+z+\cdots+z^{M-1} \bmod \left(1-z^{M}\right) .
$$

Note that $a(1)=\hat{a}(1)=m, b(1)=\mu, c(1)=\nu$ and $\left(1-z^{M}\right)_{z=1}=0$. So putting $z=1$ into the two congruences immediately above gives

$$
\begin{aligned}
& m \nu+\mu=M, \\
& m \mu+\nu=M .
\end{aligned}
$$

Subtracting these equations gives $(m-1)(\nu-\mu)=0$. Since $m \neq 1$ by assumption we deduce $\nu=\mu$ and $M=\mu(m+1)$ as required. 


\section{$5 \quad$ Extending labellings of $H$ to $H$-burrs}

In this section $H$ is a finite graph with $m$ vertices, $m>1$. We suppose that $H$ has an injective edge-magic $\mathbb{Z}$-labelling, and we derive necessary and sufficient conditions for this to be extendible to an edge-magic $\mathbb{Z}$-labelling of $G=H+\mathcal{I}$.

For integers $a \leq b$ let $[a, b]$ be the set $\{a, a+1, \cdots, b\}$ consisting of the $b-a+1$ integers lying between $a$ and $b$ inclusive. We call such a set an interval of integers. If we assume $H_{V}=[a, b]$ then $m=b-a+1$. Since $m>1$, it follows that $b>a$.

Theorem 23. Suppose we have an injective edge-magic $\mathbb{Z}$-labelling of a finite graph $H$ with edge-magic constant $k$ such that $H_{V}=[a, b]$ with $b>a$, i.e $m=\left|H_{V}\right|>1$.

We can extend this to an edge-magic $\mathbb{Z}$-labelling of $G=H+\mathcal{I}$ if and only if one of the following holds.

(1) $k<3 a-2$ and $H_{E}=\{k-a-b\}$.

(2) $k>3 b+2$ and $H_{E}=\{k-a-b\}$.

(3) $3 a \leq k \leq 2 a+b,\left|H_{E}\right|=2 a+b-k$ and if $2 a+b-k>0$ then $H_{E}=[k-a-b, a-1]$.

(4) $a+2 b \leq k \leq 3 b,\left|H_{E}\right|=k-a-2 b$ and if $k-a-2 b>0$ then $H_{E}=[b+1, k-a-b]$.

In each case the extension to an edge-magic $\mathbb{Z}$-labelling of $G$ is unique up to permutation of the labels of $\mathcal{I}$.

In cases (1) and (2) the extension is given by taking

$$
I_{V}=\{k-a-b+t(m+1) \mid t= \pm 1, \pm 2, \cdots\} .
$$

In case (3) the extension is given by taking

$$
I_{V}=\{(a-1)+t(m+1) \mid t=1,2, \cdots\} \cup\{k-a-b+t(m+1) \mid t=-1,-2, \cdots\} .
$$

In case (4) the extension is given by taking

$$
I_{V}=\{(k-a-b+t(m+1) \mid t=1,2, \cdots\} \cup\{b+1+t(m+1) \mid t=-1,-2, \cdots\} .
$$

Before proving this theorem, first let us establish some preliminary results assuming we have an edge-magic $\mathbb{Z}$-labelling of $H+\mathcal{I}$ with edge constant $k$ and $H_{V}=[a, b]$.

Lemma 24. The set $B_{E}$ is the disjoint union of the intervals $[k-\nu-b, k-\nu-a], \nu \in I_{V}$.

Proof. Consider the labels of the $m$ edges from the vertex of $\mathcal{I}$ labelled $\nu$.

Lemma 25. If $\nu^{\prime}>\nu$ and $\nu, \nu^{\prime} \in I_{V}$ then $\nu^{\prime}-\nu>b-a$.

Proof. If $\nu^{\prime}>\nu$ and $\nu, \nu^{\prime} \in I_{V}$ then $[k-\nu-b, k-\nu-a] \subset B_{E}$ and $\left[k-\nu^{\prime}-b, k-\nu^{\prime}-a\right] \subset B_{E}$ are disjoint intervals with $k-\nu-b<k-\nu^{\prime}-b$. Hence $k-\nu-a<k-\nu^{\prime}-b$ i.e. $\nu^{\prime}-\nu>b-a$. 
Definition 26. Set

$$
\nu^{*}=k-a-b .
$$

This integer, which occurs already in the statement of Theorem 23 is significant because

$$
H_{V}=[a, b]=\left[k-\nu^{*}-b, k-\nu^{*}-a\right] .
$$

Note that $\nu^{*}$ is the midpoint of $[k-2 b, k-2 a]$.

Lemma 29. The intersection of $[k-2 b, k-2 a]$ and $I_{V}$ is empty.

Proof. Let $\nu \in I_{V}$. Then the interval $[k-\nu-b, k-\nu-a] \subset B_{E}$, must be disjoint from $H_{V}=\left[k-\nu^{*}-b, k-\nu^{*}-a\right]$. Hence as in Lemma 25 if $\nu>\nu^{*}$, then $\nu-\nu^{*}>b-a$ or equivalently $\nu>k-2 a$ and similarly if $\nu<\nu^{*}$, we have $\nu<k-2 b$.

Lemma 30. The integer $\nu^{*} \in H_{V} \cup H_{E}$.

Proof. Since $\nu^{*} \in[k-2 b, k-2 a]$, it follows from Lemma 29 that $\nu^{*} \notin I_{V}$.

It remains to show that $\nu^{*} \notin B_{E}$. To have $\nu^{*} \in B_{E}$ there would have to be a $\nu \in I_{V}$ such that $\nu^{*} \in[k-\nu-b, k-\nu-a]$. However $k-\nu-b \leq k-a-b=\nu^{*}$ if and only $\nu \geq a$ and $\nu^{*}=k-a-b \leq k-\nu-a$ if and only if $\nu \leq b$ and so $\nu^{*} \in[k-\nu-b, k-\nu-a]$ if and only if $\nu \in[a, b]=H_{V}$, which is disjoint from $I_{V}$. So $\nu^{*} \notin B_{E}$.

Lemma 31. The set of integers $H_{E} \subseteq[k-2 b+1, k-2 a-1]$.

Proof. The extreme values for elements of $H_{E}$ are given by the label on an edge with vertices labelled by $b$ and $b-1$ or by $a$ and $a+1$. Thus any element of $H_{E}$ must lie between $k-b-(b-1)=2 b+1$ and $k-a-(a+1)=k-2 a-1$.

Lemma 32. (1) The labels $k-2 b, k-2 a \in H_{V} \cup B_{E}$.

(2) At least one of $k-2 b$ and $k-2 a$ is an element of $B_{E}$.

(3) The integer $k-2 b \in B_{E}$ if and only if $k-2 b \in\left[k-\nu_{b}-b, k-\nu_{b}-a\right] \subset B_{E}$ for some $\nu_{b} \in I_{V}$ satisfying

$$
b+1 \leq \nu_{b} \leq b+(b-a)
$$

(4) The integer $k-2 a \in B_{E}$ if and only if $k-2 a \in\left[k-\nu_{a}-b, k-\nu_{a}-a\right] \subset B_{E}$ for some $\nu_{a} \in I_{V}$ satisfying

$$
a-(b-a) \leq \nu_{a} \leq a-1 .
$$


Proof. From Lemmas 29 and 31 neither $k-2 b$ nor $k-2 a$ is an element of $I_{V} \cup H_{E}$. The first assertion follows.

Since $(k-2 a)-(k-2 b)=2(b-a)>b-a$ at most one of $k-2 b$ and $k-2 a$ can lie in the interval $[a, b]$. Thus they cannot both be elements of $H_{V}$. The second assertion follows.

The integer $k-2 b \in B_{E}$ if and only if $k-2 b \in\left[k-\nu_{b}-b, k-\nu_{b}-a\right]$ for some $\nu_{b} \in I_{V}$, i.e. $k-\nu_{b}-b \leq k-2 b \leq k-\nu-a$, i.e.

$$
b \leq \nu \leq 2 b-a=b+(b-a)
$$

But $\nu_{b} \notin H_{V}$ implies that $\nu_{b} \neq b$. Hence $k-2 b \in B_{E}$ if and only if there exists $\nu_{b} \in I_{V}$ with

$$
b+1 \leq v_{b} \leq b+(b-a)
$$

Hence the third assertion.

A similar argument proves the fourth assertion.

Suppose we have a set of disjoint intervals. Then they come naturally ordered: $[c, d]<$ $[e, f]$ if and only if $d<e$. Call two intervals of integers $[c, d],[e, f]$ adjacent if there is no gap between them, that is $e=d+1$ or $c=f+1$.

Lemma 36. (1) The set $B_{E} \cup H_{V}$ is the union of disjoint intervals

$$
\left\{[k-b-\nu, k-a-\nu] \mid \nu \in I_{V} \cup\left\{\nu^{*}\right\} .\right.
$$

Each consists of $m=(b-a)+1$ consecutive integers.

(2) The integers not in such an interval are elements of $H_{E} \cup I_{V}$.

(3) Two consecutive intervals of $B_{E} \cup H_{V}$ are adjacent (not separated by an element of $\left.H_{E} \cup I_{V}\right)$ if and only if they are of the form $[k-\nu-b, k-\nu-a]$ and $\left[k-\nu^{\prime}-b, k-\nu^{\prime}-a\right]$ for a pair of integers $\nu>\nu^{\prime} \in I_{V} \cup\left\{\nu^{*}\right\}$ with $\nu-\nu^{\prime}=b-a+1$. In this case the $b-a-1$ integers strictly between $\nu$ and $\nu^{\prime}$ are elements of $H_{E}$.

Proof. The first statement follows from Lemma 24 and equation (28). For the second assertion assume $[k-\nu-b, k-\nu-a]$ and $\left[k-\nu^{\prime}-b, k-\nu^{\prime}-a\right]$ are adjacent. This is the case if and only if $\left|\nu-\nu^{\prime}\right|=(b-a)+1$. By Lemma 25 there is no room for any elements of $I_{V}$ between $\nu$ and $\nu^{\prime}$. Also $\left|\nu-\nu^{\prime}\right|=(b-a)+1$ implies there are only $b-a$ integers strictly between $\nu$ and $\nu^{\prime}$ and no elements of $B_{E} \cup H_{V}$ can lie between them as such labels come in intervals of $(b-a)+1$ integers.

We are now ready to complete the proof of Theorem 23.

Proof of Theorem 23. Case 1: Suppose that $k-2 b, k-2 a \in B_{E}$. By Lemma 32, neither $k-2 b$ nor $k-2 a$ lies in $[a, b]$. By Lemma 24, $k-2 b \in\left[k-\nu_{b}-b, k-\nu_{b}-a\right]$ for some $\nu_{b} \in I_{V}$ satisfying (33) and $k-2 a \in\left[k-\nu_{a}-b, k-\nu_{a}-a\right]$ for some $\nu_{a} \in I_{V}$ 
satisfying (34). Moreover, one of $(k-2 a<a),(k-2 b<a$ and $k-2 a>b)$ or $(k-2 b>b)$ must hold, i.e.we have the following subcases: $k<3 a$ (Case 1A) or $a+2 b<k<2 b+a$ (Case 1B) or $k>3 b$ (Case 1C).

We first eliminate Case 1B: $a+2 b<k<2 b+a$.

In this case the interval $J=\left[k-\nu_{b}-b, k-\nu_{b}-a\right] \subseteq B_{E}$ containing $k-2 b<a$ must be to the left of the interval $[a, b]$. This interval $J$ does not contain $\nu_{a} \in I_{V}$. Any integer $\nu<k-\nu_{b}-b$ will satisfy $\nu<a-(b-a)$. For (34) of Lemma 32 to hold we must therefore have $k-\nu_{b}-a<\nu_{a}$. This implies

$$
k-a<\nu_{a}+\nu_{b}
$$

Similarly the interval $\left[k-\nu_{a}-b, k-\nu_{a}-a\right]$ containing $k-2 a$ must be to the right of the interval $[a, b]$ and it follows similarly that $k-\nu_{a}-b>\nu_{b}$ which implies

$$
k-b>\nu_{a}+\nu_{b} .
$$

But (37) and (38) together imply $a>b$, a contradiction to our assumption $b>a$.

Hence if both $k-2 a$ and $k-2 b$ are elements of $B_{E}$ we must have either $k-2 a<a$, i.e $k<3 a$ or $k-2 b>b$, i.e. $k>3 b$.

Consider now Case 1A: $k<3 a$.

In this case $k-2 a-1<a-1$. So by Lemma 31 there are no elements of $H_{E}$ greater than or equal to $a-1$. In particular $a-1 \notin H_{E}$. If $a-1 \in B_{E}$, then from Lemma 36, $k-\nu-b=a-1$ for some $\nu \in K$ with $\left|\nu-\nu^{*}\right|=b-a+1$. But then $\nu=k-a-b+1$ and $\nu^{*}=k-a-b$ contradict the fact that $b-a+1 \geq 2$. Thus we must have $\nu_{a}=a-1$ and $\nu_{b}=b+1$ by Lemma 32 .

Let $J_{b}=[k-2 b-1, k-a-1]$ and $J_{a}=[k-a-b+1, k-2 a+1]$ be the intervals in $B_{E}$ associated to $\nu_{b}=b+1$ and $\nu_{a}=a-1$ respectively. They are are both to the left of $[a, b]$ since $k-2 a<a$ lies in the right most $J_{a}$, which is disjoint from $[a, b]$. The intervals $J_{a}$ and $J_{b}$ are separated by the single integer $k-a-b=\nu^{*}$. Bearing in mind Lemma 31, we have that $H_{E}=\left\{\nu^{*}\right\}$. Lemma 36 implies now that all other pairs adjacent blocks are separated by a single integer from $I_{V}$. We must also have $k-2 a+2 \in I_{V}$. Hence $k-2 a+2 \leq \nu_{a}=a-1$, i.e $k \leq 3 a-3$.

Then the labels making up $I_{V} \cup\left\{\nu^{*}\right\}$ are separated by intervals of $m=(b-a)+1$ integers. Hence these labels are congruent modulo $m+1$ and so make up the set of integers congruent to $\nu^{*}=k-a-b$ modulo $m+1$. Thus the set of labels of the vertices in $\mathcal{I}$, $I_{V}=\{k-a-b+t(m+1) \mid t \neq 0\}$.

A similar argument holds for Case 1C: $k>3 b$, and gives again $H_{E}=\left\{\nu^{*}\right\}$ and $I_{V}=\{k-a-b+t(m+1) \mid t \neq 0\}$.

It remains now to consider Case 2, in which one of $k-2 a$ and $k-2 b$ is an element of $H_{V}$. By Lemma 32 the other lies in $B_{E}$. Note $k-2 a \in H_{V}$ if and only if $3 a \leq k \leq 2 a+b$ and $k-2 b \in H_{V}$ if and only if $a+2 b \leq k \leq 3 b$.

Case 2A: Suppose that $3 a \leq k \leq 2 a+b$. Then $k-2 b \in B_{E}$. So Lemma 32 implies there exists $\nu_{b} \in I_{V}$ satisfying (33). We show that $\nu_{b}=b+1$.

By the first part of Lemma 36 if $\nu_{b}>b+1$ there must be an element of $H_{E}$ greater than $b$. From $k-2 a \leq b$ we deduce $k-2 a-1<b$ which together with Lemma 31 implies 
there are no elements of $H_{E}$ greater than $b$. We must therefore have $\nu_{b}=b+1$ as required. Also as $H_{E} \cap[a, b]=\emptyset$, any $\nu \in H_{E}$ must satisfy $\nu<a$ (needed below).

We now show

$$
H_{E}=\{\nu \mid k-a-b-1<\nu<a\} .
$$

The interval $\left[k-\nu_{b}-b, k-\nu_{b}-a\right]=[k-2 b-1, k-a-b-1] \subseteq B_{E}$ and it lies to the left of $[a, b]$. Since $k-2 b-1<k-2 b+1$ from Lemma 31 we deduce that any $\nu \in E_{H}$ has $\nu>k-a-b-1$. Thus any elements of $H_{E}$ lie strictly between $k-a-b-1$ and $a$. To establish (39) it remains to show that every integer strictly between $k-a-b-1$ and $a$ lies in $H_{E}$. Since $k \geq 3 a, k-a-b \geq 2 a-b=a-(b-a)$. So by Lemma 32, as $k-2 a \notin B_{E}$, there are no elements of $I_{V}$ strictly between $k-a-b-1$ and $a$. Hence all such integers are elements of $H_{E}$ by Lemma 36 .

Note if $k=2 a+b$ then $k-a-b=a$ and $E_{H}=\emptyset$, this implies $H$ has no edges, whereas if $3 a \leq k<2 a+b,\left|E_{H}\right|=2 a+b-k$ and $E_{H}=[k-a-b, a-1]=\left[\nu^{*}, a-1\right]$, is an interval and so is the complete set of labels of the graph elements of $H$,

$$
H_{V} \cup H_{E}=[k-a-b, b] .
$$

Having established (39), we have that the interval of $B_{E}$ containing $k-b$ lies to the left of $[a, b]$ and the intervening integers form the set $E_{H}$. Hence Lemma 36 implies all consecutive pairs of intervals in $B_{E} \cup H_{V}$ except that containing $k-2 b$ and $[a, b]$ are separated by a single element of $I_{V}$.

If we order the elements of $I_{V}$ greater than $\nu^{*}$ then consecutive labels are separated by a block of $m=(b-a)+1$ integers. These elements are congruent modulo $(b-a)+2=m+1$ and form the half-modulus class

$$
\{(b+1)+t(m+1) \mid t \geq 0\}=\{(a-1)+t(m+1) \mid t>0\} .
$$

Similarly the elements of $I_{V}$ less than $\nu^{*}$ form the half-modulus class

$$
\{k-a-b+t(m+1) \mid t<0\} .
$$

Case 2B: Suppose lastly $2 b+a \leq k \leq 3 b$.

A similar argument to the above shows

$$
H_{E}=\{\nu \mid k-a-b-1<\nu<a\} .
$$

so that for $k=a+2 b, H_{E}=\emptyset, a+2 b \leq k \leq 3 b$ and $2 b+a-k>0, H_{E}=\left[b+1, \nu^{*}\right]$. Hence in the case $2 b+a \leq k \leq 3 b$, the labels of the graph elements of $H$,

$$
H_{V} \cup H_{E}=[a, k-a-b] .
$$

Further the elements of $I_{V}$ greater than $\nu^{*}$ form the half-modulus class

$$
\{k-a-b+t(m+1) \mid t>0\},
$$


and elements of $I_{V}$ less than $\nu^{*}$ form the half-modulus class

$$
\{a-1+t(m+1) \mid t \leq 0\}=\{b+1+t(m+1) \mid t<0\}
$$

The necessary conditions of the theorem have now been established. When these hold we can extend the injective labelling on $H$ to an edge-magic labelling of $G$, by labelling the vertices $\mathcal{I}$ by the given half-modulus classes.

Corollary 43. If $H$ has exactly one edge then $H+\mathcal{I}$ has an edge-magic $\mathbb{Z}$-labelling.

Proof. Immediate.

Recall [7] that a finite graph $H$ with $m$ edges and $\ell$ vertices is called edge magic, with edge constant $k$ if there a bijective labelling of the graph elements of $H$ by the integers $[1, m+\ell]$ which gives each edge weight $k$. This labelling is called strong if the labels on the vertices form the interval of integers $[1, m]$.

Corollary 44. Suppose $H$ has more than one edge. Then $H+\mathcal{I}$ has an edge-magic $\mathbb{Z}$ labelling (in which the vertices of $H$ are labelled by consecutive integers) if and only $H$ has a strong edge-magic labelling, with edge constant $k$ satisfying

$$
2 m+3 \leq k \leq 3 m
$$

Proof. Suppose $H$ has more than one edge and $H+\mathcal{I}$ has an edge-magic $\mathbb{Z}$-labelling with constant $k$ for which $H_{V}=[a, b]$. Then either (3) or (4) of Theorem 23 holds. But if (3) holds, then replacing every label $n$ by $(a+b)-n$ gives an edge-magic $\mathbb{Z}$-labelling of $H$ with edge constant $3(a+b)-k$ for which (4) holds. So without loss of generality there exists an edge-magic $\mathbb{Z}$-labelling with constant $k$, satisfying $a+2 b+2 \leq k \leq 3 b$. Note we cannot have $k=a+2 b$, or $k=a=2 b+1$ because these give $\ell=\left|H_{E}\right| \leq 1$. By (4), recalling (42), we have a total labelling of the graph elements of $H$ by $[a, k-a-b]$, in which the vertices of $H$ are labelled by $[a, b]$. Subtracting $a-1$ from each label gives a strong edge-magic labelling of $H$ with edge-constant $k^{\prime}=k-3 a+3$, with $2(b-a)+5 \leq k^{\prime} \leq 3(b-a)+3$. But $m=(b-a)+1$. Hence $H$ has a strong edge-magic labelling where the edge-constant $k^{\prime}$ satisfies $2 m+3 \leq k^{\prime} \leq 3 m$. Reversing the above shows the converse.

Corollary 45. Suppose $H+\mathcal{I}$ has an edge-magic $\mathbb{Z}$-labelling in which the vertices of $H$ are labelled by consecutive integers. Then $H$ has fewer edges than vertices.

Proof. Suppose $H+\mathcal{I}$ has such labelling with edge-constant $k$.

If $H$ has $m \leq 2$ vertices the number of edges is less than the number of vertices. The result is trivially true.

If $H$ has $m \geq 3$ vertices apply the theorem. In case (1) or (2) above then $H$ has only one edge and the result is trivially true. In case (3) note that $3 a \leq k$ is equivalent to $2 a+b-k \leq b-a=m-1$. Thus in case (3) the number of edges of $H,\left|H_{E}\right|=2 a+b-k<m$.

Similarly in case (4) the number of edges of $H,\left|H_{E}\right|<m$. 
Example 46. Let $H=T_{m-1}$ be a star on $m$ vertices. Then there is an edge-magic $\mathbb{Z}$-labelling of the $H+\mathcal{I}$ with edge constant 0 .

Proof. Let $H_{V}=\{0,1,2, \ldots, m-1\}$ (with the centre of the star labelled with 0 ) so that $H_{E}=\{-1,-2, \ldots,-(m-1)\}$. Then we have Case 3 of Theorem 23 with $a=k=0$, $b=m-1$. Thus

$$
I_{V}=\{-1+t(m+1) \mid t=1,2, \ldots\} \cup\{-m+1+t(m+1) \mid t=-1,-2, \ldots\},
$$

with $B_{E}=\mathbb{Z} \backslash\left(I_{V} \cup H_{V} \cup H_{E}\right)$.

If we relax the assumption that $H_{V}$ is a set of consecutive integers, the problem considered in this section becomes much more complicated. However, the following lemma shows a special case.

Lemma 47. Let $H$ be a finite graph with $2 M \geq 2$ vertices and $2 L \geq 0$ edges and suppose there is an injective $\mathbb{Z}$-labelling of $H$ in which the vertices are labelled with the set $\{ \pm i \mid$ $1 \leq i \leq M\}$ and the edges with the set $\{ \pm i \mid M+1 \leq i \leq M+L\} \cup\{0\}$, so that the edge constant is 0 . Then we can always extend the labelling of $H$ to an edge-magic $\mathbb{Z}$-labelling of $H+\mathcal{I}$ with edge constant 0 .

Proof. Label the vertices of $\mathcal{I}$ with $\{i(2 M+2 L+1) \mid i \neq 0\}$. This forces the edges between $H$ and $\mathcal{I}$ to use all remaining $\mathbb{Z}$-labels.

Example 48. Using the previous lemma with $M=3, L=2$ and $H=P_{5}$ labelled as in Figure 2, there exists an edge-magic $\mathbb{Z}$-labelling of the $P_{5}$-burr.

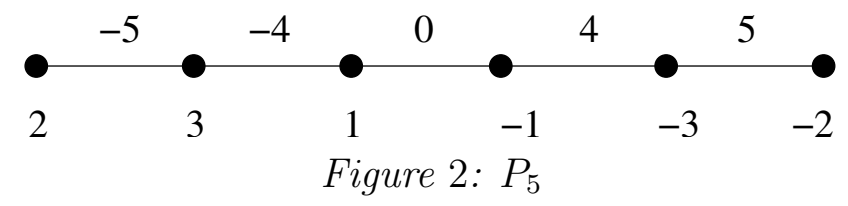

We conclude with the following open problem:

Does there exist an edge-magic $\mathbb{Z}$-labelling of $K_{3}+\mathcal{I}$ ?

Note that by Corollary 45 there is no such labelling in which the vertices of $K_{3}$ are labelled with consecutive integers.

\section{References}

[1] A.F. Beardon, Magic labellings of infinite graphs, Austral. J. Combin., 30, (2004), $117-132$.

[2] D. Combe, A.M. Nelson, W.D. Palmer, Magic labellings of graphs over finite abelian groups, Austral. J. Combin., 29, (2004), 259-271. 
[3] D. Combe, A.M. Nelson, Magic labellings of infinite graphs over infinite groups, Austral. J. Combin., 35, (2006), 193-210.

[4] J.A. Gallian, A Dynamic Survey of Graph Labeling, The Electronic Journal of Combinatorics, (October, 2002), \#DS6.

[5] R.L. Graham, M. Grøtschel, L. Lovasz, Handbook of Combinatorics, 1995.

[6] G. Pólya and G. Szegö, Problems and Theorems in Analysis II republished 1997 by Springer.

[7] W.D. Wallis, Magic Graphs, Birkhäuser, Boston, 2001 